\title{
Nucleon strange electromagnetic form factors
}

\author{
C. Alexandrou $\odot,{ }^{1,2}$ S. Bacchio, ${ }^{2}$ M. Constantinou, ${ }^{3}$ J. Finkenrath, ${ }^{2}$ K. Hadjiyiannakou, ${ }^{2}$ K. Jansen, ${ }^{4}$ and G. Koutsou ${ }^{2}$ \\ ${ }^{1}$ Department of Physics, University of Cyprus, P.O. Box 20537, 1678 Nicosia, Cyprus \\ ${ }^{2}$ Computation-based Science and Technology Research Center, The Cyprus Institute, \\ 20 Kavafi Str., Nicosia 2121, Cyprus \\ ${ }^{3}$ Department of Physics, Temple University, \\ 1925 North, 12th Street, Philadelphia, Pennsylvania 19122-1801, USA \\ ${ }^{4}$ NIC, DESY, Platanenallee 6, D-15738 Zeuthen, Germany
}

(Received 2 October 2019; accepted 21 January 2020; published 13 February 2020)

\begin{abstract}
We determine the strange electromagnetic form factors of the nucleon within the lattice formulation of quantum chromodynamics using simulations that include light, strange and charm quarks in the sea all tuned to their physical mass values. We employ state-of-the-art techniques to accurately extract the form factors for values of the momentum transfer square up to $0.8 \mathrm{GeV}^{2}$. We find that both the electric and magnetic form factors are statistically nonzero. We obtain for the strange magnetic moment $\mu^{s}=-0.017(4)\left(\begin{array}{c}+1 \\ 0\end{array}\right)\left(\begin{array}{c}+5 \\ 0\end{array}\right)$, the strange magnetic radius $\left\langle r_{M}^{2}\right\rangle^{s}=-0.015(9)\left(\begin{array}{c}+3 \\ 0\end{array}\right)\left(\begin{array}{c}+4 \\ 0\end{array}\right) \mathrm{fm}^{2}$, and the strange charge radius $\left\langle r_{E}^{2}\right\rangle^{s}=-0.0048(6)\left({ }_{-8}^{0}\right)\left(\begin{array}{c}+12 \\ 0\end{array}\right) \mathrm{fm}^{2}$, where the first error is statistical, and the second and third systematic.
\end{abstract}

DOI: 10.1103/PhysRevD.101.031501

\section{INTRODUCTION}

Strange quarks are the lightest nonvalance quarks in the nucleon and thus the most likely constituents to contribute to sea-quark dynamics. The study of strange-quark contributions to nucleon structure observables allows us to uniquely identify sea-quark effects and understand virtual particle dynamics in the nonperturbative regime of quantum chromodynamics (QCD). A possible difference in the spatial distribution of strange and antistrange quarks reflected by a nonzero strange electric form factor $G_{E}^{s}\left(Q^{2}\right)$ and a finite strange magnetic moment $\mu^{s} \equiv G_{M}^{s}\left(Q^{2}=0\right)$ are key quantities describing the nontrivial composite structure of the nucleon. Parity-violating electron-proton elastic scattering events probing the interference of photons and Z-bosons exchanges enable the measurement of the strange form factors and weak charge of the proton [1]. An accurate determination of the neutral-weak vector form factor in combination with the electromagnetic form factors of the nucleon are needed in order to put constraints on new physics beyond the standard model.

Theoretical studies of the strange form factors of the proton using various models have a long history [2-10]. They are complemented by major experiments. The experimental program to study the strangeness in the proton

Published by the American Physical Society under the terms of the Creative Commons Attribution 4.0 International license. Further distribution of this work must maintain attribution to the author(s) and the published article's title, journal citation, and DOI. Funded by SCOAP ${ }^{3}$. began with the SAMPLE experiment $[11,12]$ and it has been continuing with the series of A4 experiments at the Mainz Microtron accelerator facility [13-15], and the HAPPEX [16-19] and G0 experiments [20,21] at JLab. However to date, the experimental results, although indicating nonzero values, carry large errors that make them inconclusive. This is confirmed by a recent global analysis of parity-violating elastic scattering data [22], where, although a negative magnetic strange form factor is indicated, the large error still makes it consistent with zero. A review of the experimental program and results can be found in Ref. [23].

Given the current status of the experimental results, where there is no agreement even on the sign of the strange electromagnetic form factors, a first principle calculation of these key quantities is crucial. Lattice QCD provides a rigorous framework to compute nonperturbatively these quantities. However, it is only recently that efficient algorithms have enabled simulations of the theory with physical values of the light quark masses and the evaluation of disconnected quark loops to sufficient accuracy [24-27]. In this work, we use simulations generated with physical values of the light quark masses to evaluate accurately the strange-quark loops and extract the strange electromagnetic form factors directly at the physical point.

\section{LATTICE METHODOLOGY}

The results of this work are based on the analysis of an ensemble simulated with two mass degenerate light quarks, a strange and a charm quark denoted by $N_{f}=2+1+1$, with masses tuned to their physical values [28] within the 
twisted mass formulation [29-31] including a clover term [32]. The lattice volume is $64^{3} \times 128, m_{\pi} L=3.62$, where $L$ is the spatial lattice length, the pion mass $m_{\pi}=$ $0.1393(7) \mathrm{MeV}$ and the lattice spacing $a=0.0801$ (4) fm, determined from the nucleon mass [33]. We will refer to this ensemble as the cB211.072.64 ensemble. We use Osterwalder-Seiler strange and charm quarks with mass tuned to reproduce the $\Omega^{-}$baryon mass and the mass of $\Lambda_{c}$, respectively.

The nucleon matrix element of the electromagnetic (EM) current $j_{\mu}^{s}$ decomposes into two $C P$-even form factors given by

$$
\left\langle N\left(p^{\prime}, s^{\prime}\right)\left|j_{\mu}^{s}\right| N(p, s)\right\rangle \propto \bar{u}_{N}\left(p^{\prime}, s^{\prime}\right) \Lambda_{\mu}^{s}\left(q^{2}\right) u_{N}(p, s)
$$

with

$$
\Lambda_{\mu}^{s}\left(q^{2}\right)=\gamma_{\mu} F_{1}^{s}\left(q^{2}\right)+\frac{i \sigma_{\mu \nu} q^{\nu}}{2 m_{N}} F_{2}^{s}\left(q^{2}\right)
$$

where $F_{1}^{s}\left(q^{2}\right), F_{2}^{s}\left(q^{2}\right)$ are the Dirac and Pauli form factors for the strange quark $s, N(p, s)$ is the nucleon state with

mass $m_{N}$, momentum $p$, spin $s$ and energy $E_{N}(\vec{p}) \cdot u_{N}$ is the nucleon spinor, $q^{2}=\left(p_{\mu}^{\prime}-p_{\mu}\right)^{2}$ is the square of the momentum transfer and $j_{\mu}^{s}=e_{s} \bar{s}(x) \gamma_{\mu} s(x)$, where $e_{s}=-1 / 3$. The electric and magnetic Sachs form factors can be expressed as linear combinations of the Dirac and Pauli form factors given in Euclidean space via the relations,

$$
\begin{gathered}
G_{E}^{s}\left(Q^{2}\right)=F_{1}^{s}\left(Q^{2}\right)-\frac{Q^{2}}{4 m_{N}^{2}} F_{2}^{s}\left(Q^{2}\right) \\
G_{M}^{s}\left(Q^{2}\right)=F_{1}^{s}\left(Q^{2}\right)+F_{2}^{s}\left(Q^{2}\right)
\end{gathered}
$$

where $Q^{2}=-q^{2}$. In order to extract the electric and magnetic strange form factors $G_{E}^{s}$ and $G_{M}^{s}$ we evaluate the two- and three-point correlation functions, given by

$$
C\left(\Gamma_{0}, \vec{p} ; t_{s}\right)=\sum_{\vec{x}_{s}} \operatorname{Tr}\left[\Gamma_{0}\left\langle J_{N}\left(t_{s}, \vec{x}_{s}\right) \bar{J}_{N}(0, \overrightarrow{0})\right\rangle\right] e^{-i \vec{x}_{s} \cdot \vec{p}}
$$

$$
C_{\mu}^{s}\left(\Gamma_{\nu}, \vec{q}, \vec{p}^{\prime} ; t_{s}, t_{\mathrm{ins}}\right)=\sum_{\vec{x}_{s}, \vec{x}_{\mathrm{ins}}} e^{+i \vec{x}_{\mathrm{ins}} \cdot \vec{q}-i \vec{x}_{s} \cdot \vec{p}^{\prime}} \times \operatorname{Tr}\left[\Gamma_{\nu}\left\langle J_{N}\left(t_{s}, \vec{x}_{s}\right) j_{\mu}^{s}\left(t_{\mathrm{ins}}, \vec{x}_{\mathrm{ins}}\right) \bar{J}_{N}(0, \overrightarrow{0})\right\rangle\right]
$$

$x_{0}=(0, \overrightarrow{0})$ is where a state with the nucleon quantum numbers is created (source), $x_{s}$ where the same state is annihilated (sink) and $x_{\text {ins }}$ denotes the lattice site at which the current couples to a quark. We use projectors $\Gamma_{\nu}$ taking for the unpolarized $\Gamma_{0}=\frac{1}{2}\left(1+\gamma_{0}\right)$ and for the polarized $\Gamma_{k}=\Gamma_{0} i \gamma_{5} \gamma_{k} . J_{N}(x)=\epsilon^{a b c} u^{a}(x)\left[u^{b T}(x) \mathcal{C} \gamma_{5} d^{c}(x)\right]$, is the standard interpolating field for the nucleon, and $\mathcal{C}$ is the charge conjugation matrix. We use Gaussian smearing $[34,35]$ for the quark interpolating fields with APEsmeared [36] gauge links in the hopping operator with parameters given in Ref. [33].

The EM current $j^{s}$ couples to a sea strange quark and the contribution of the strange-quark loop is given by

$$
\sum_{\vec{x}_{\text {ins }}} e^{+i \vec{q} \cdot \vec{x}_{\text {ins }}} \operatorname{Tr}\left[\gamma^{\mu} G\left(x_{\text {ins }} ; x_{\text {ins }}\right)\right] .
$$

We use stochastic approaches combined with dilution methods [37] to compute such quark loops [38]. Namely, we perform a full dilution in spin and color [39] to avoid any stochastic contamination in that subspace.
The elements of the propagator decay exponentially with the distance $|x-y|$ thus dilution in space-time up to a specific distance reduces stochastic contamination entering from off-diagonal elements. This is implemented by employing the hierarchical probing technique [40] using a four dimensional coloring of distance- $2^{3}$ resulting in $N_{\text {Had }}=512$ Hadamard vectors. We also exploit properties of the twisted mass fermions in the so-called one-end trick [41,42], which improves signal-to-noise ratio [43,44]. We compute the quark loops for every time slice $t_{\text {ins }}$ and construct the disconnected three-point function at every value of $t_{s}$ and $t_{\text {ins }}$ by correlating it with 200 nucleon twopoint functions of randomly distributed source positions per gauge configuration. We use 750 configurations, averaging over proton and neutron and forward and backward propagators to reach in total 600,000 measurements. We utilize the multigrid algorithm implemented on GPUs through the QUDA software [45-47] to accelerate the calculation of the quark propagators.

The nucleon matrix element is extracted from an optimally constructed ratio [48-50] given by

$$
R_{\mu}\left(\Gamma_{\nu}, \vec{p}^{\prime}, \vec{p} ; t_{s}, t_{\mathrm{ins}}\right)=\frac{C_{\mu}\left(\Gamma_{\nu}, \vec{p}^{\prime}, \vec{p} ; t_{s}, t_{\mathrm{ins}}\right)}{C\left(\Gamma_{0}, \vec{p}^{\prime} ; t_{s}\right)} \times \sqrt{\frac{C\left(\Gamma_{0}, \vec{p} ; t_{s}-t_{\mathrm{ins}}\right) C\left(\Gamma_{0}, \vec{p}^{\prime} ; t_{\mathrm{ins}}\right) C\left(\Gamma_{0}, \vec{p}^{\prime} ; t_{s}\right)}{C\left(\Gamma_{0}, \vec{p}^{\prime} ; t_{s}-t_{\mathrm{ins}}\right) C\left(\Gamma_{0}, \vec{p} ; t_{\mathrm{ins}}\right) C\left(\Gamma_{0}, \vec{p} ; t_{s}\right)}},
$$


which becomes time independent for $\Delta E\left(t_{s}-t_{\text {ins }}\right) \gg 1$ and $\Delta E t_{\text {ins }} \gg 1$ yielding $\Pi_{\mu}\left(\Gamma_{\nu}, \vec{p}^{\prime}, \vec{p}\right)$, where $\Delta E$ is the energy gap between the ground and the first excited state. We employ three methods to check the convergence to the ground state matrix element [38,51]: (i) Plateau method: We use the ratio of Eq. (8) and identify a time-independent window (plateau), where we fit to a constant. We seek convergence of this value as we increase $t_{s}$. (ii) Two-state fit: Takes into account the first excited state in the three- and two-point correlators entering in the ratio of Eq. (8). More details are given in Ref. [38]. (iii) Summation method: [52] Summing over the insertion time $t_{\text {ins }}$ in Eq. (8), excluding contact terms, leads to a linear behavior. The slope yields the nucleon state matrix element as $t_{s}$ increases. For all the three methods we use correlated $\chi^{2}$ fits that take into account correlations between different insertion time slices and source-sink time separations.

For disconnected quantities we are not limited to using $\vec{p}^{\prime}=\overrightarrow{0}$ since no additional inversions are needed. Given that $\vec{p}=\frac{2 \pi}{L} \vec{n}$, we analyze three-point functions as described in Ref. [33]. We take $\left|\vec{n}^{\prime}\right|^{2} \leq 2$, and $|\vec{n}|^{2} \leq 11$ for $G_{E}$ and $|\vec{n}|^{2} \leq 26$ for $G_{M}$ since the latter is in general more accurate allowing us to reach higher values of the momentum.

In Fig. 1 we show the electric and magnetic form factors for two representative values of $Q^{2}$. As can be seen, the plateau method converges as $t_{s}$ increases yielding agreement with the two-state fit and summation method as $t_{s}^{\text {low }}$ increases. We take as a systematic for excited state effects

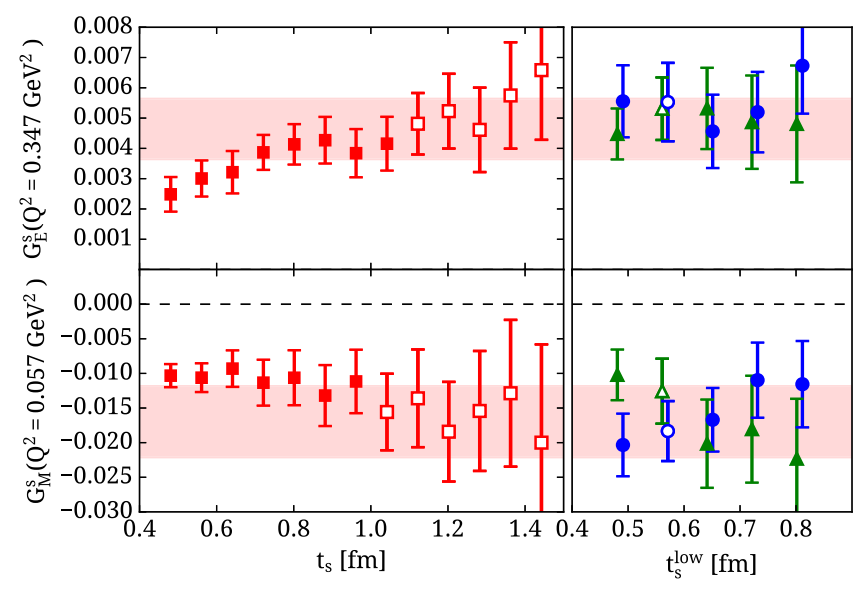

FIG. 1. The electric $G_{E}^{s}\left(Q^{2}\right)$ (upper) and magnetic $G_{M}^{s}\left(Q^{2}\right)$ (lower) form factors for $Q^{2}=0.347 \mathrm{GeV}^{2}$ and $Q^{2}=0.057 \mathrm{GeV}^{2}$, respectively. We show the extracted values using the plateau method (left) as a function of $t_{s}$ (red squares) and using the summation method (green triangles) and two-state fit (blue circles) (right) as a function of the lowest value of $t_{s}$ used in the fits, denoted by $t_{s}^{\text {low }}$. The largest value of $t_{s}$ is fixed to $t_{s}=1.44 \mathrm{fm}$ for the summation method and to $t_{s}=1.12 \mathrm{fm}$ for the two-state fits. Open symbols indicate the values of $t_{s}$ for the plateau and $t_{s}^{\text {low }}$ for the summation and two-state fits, where convergence to the ground state is reached. The weighted average of the plateau values is shown by the red band. the difference between the maximum and minimum values extracted from the plateau and the summation method. For the plateau we take weighted average of the converged values and for the summation we fit in the range of $t_{s}=0.56-1.44 \mathrm{fm}$.

\section{RENORMALIZATION}

We compute the renormalization function $Z_{V}$ by employing the Rome-Southampton method (RI'-MOM scheme) [53] and the momentum source approach introduced in Ref. [54], achieving a per mil statistical accuracy using $\mathcal{O}(10)$ configurations [55-57]. Discretization effects are suppressed using momenta that have the same spatial components, satisfying $\sum_{i} p_{i}^{4} /\left(\sum_{i} p_{i}^{2}\right)^{2}<0.3$ [58]. Furthermore, we subtract unwanted finite- $a$ effects to $\mathcal{O}\left(g^{2} a^{\infty}\right)$ using results from lattice perturbation theory [57]. We analyze five $N_{f}=4$ ensembles simulated with a range of pion masses in order to take the chiral limit. These gauge configurations are dedicatedly produced for the renormalization program using the same $\beta$ value as the $N_{f}=2+1+1$ ensemble of this work. On each ensemble we compute 25 different values of the initial renormalization scale $\left(a \mu_{0}\right)^{2} \in[1-7]$. After extrapolating to the chiral limit for each 25 values we then extrapolate to $\left(a \mu_{0}\right)^{2} \rightarrow 0$ to remove any residual dependence on the RI'-MOM scale. Due to the subtraction of the $\mathcal{O}\left(g^{2} a^{\infty}\right)$ artifacts, an almost zero slope line is obtained for $Z_{V}$ for $\left(a \mu_{0}\right)^{2} \in[2-7]$. We obtain as our final value of $Z_{V}=0.728(1)(4)$, where the first parenthesis gives the statistical error and the second the systematic coming from varying the fit window in the $\left(a \mu_{0}\right)^{2} \rightarrow 0$ extrapolation. More details can be found in Refs. [38,57,59].

\section{RESULTS}

The results for the strange electric form factor $G_{E}^{s}\left(Q^{2}\right)$ are presented in Fig. 2. The form factor is zero at $Q^{2}=0$ as expected and reaches a maximum at about $Q^{2} \simeq 0.4 \mathrm{GeV}^{2}$. In Fig. 3 we show results for the strange magnetic form factor $G_{M}^{s}\left(Q^{2}\right)$, which is clearly negative and nonzero becoming increasingly more negative as $Q^{2} \rightarrow 0$. We fit the $Q^{2}$ dependence of the form factors employing the model independent z-expansion [60-62],

$$
G\left(Q^{2}\right)=\sum_{k=0}^{k_{\max }} a_{k} z^{k}, \quad z\left(Q^{2}\right)=\frac{\sqrt{t_{\mathrm{cut}}+Q^{2}}-\sqrt{t_{\mathrm{cut}}}}{\sqrt{t_{\mathrm{cut}}+Q^{2}}-\sqrt{t_{\mathrm{cut}}}},
$$

using as $t_{\text {cut }}=\left(2 m_{K}\right)^{2}$ where $m_{K}=486(4) \mathrm{MeV}$ the kaon mass as measured for this ensemble. Since the series is expected to converge, one can truncate to a $k=k_{\max }$ and check convergence by increasing $k_{\max }$. Since $G_{E}^{s}(0)=0$ we set $a_{0}=0$. We truncate the series to $k_{\max }=5$ since including higher order terms have an insignificant effect on the fit. In order to stabilize the fit we use Gaussian priors for 


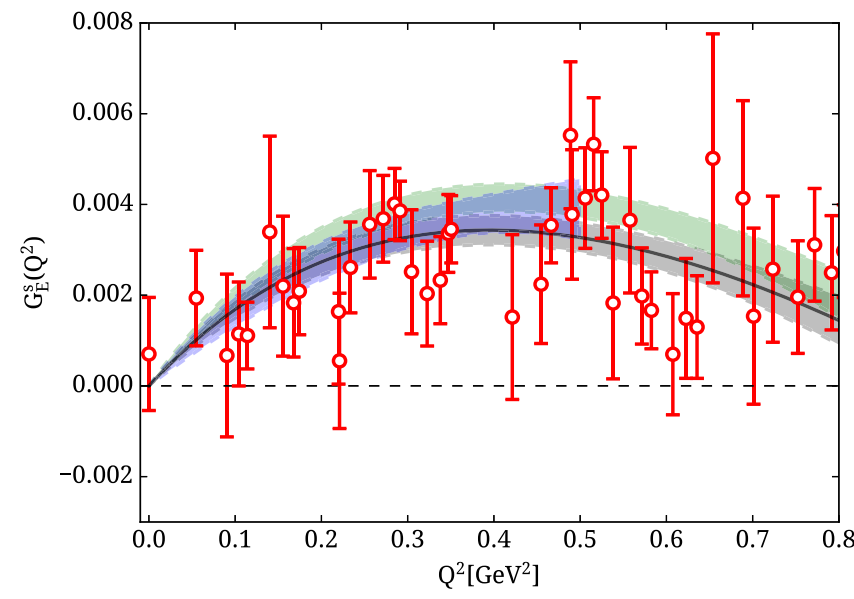

FIG. 2. $G_{E}^{s}\left(Q^{2}\right)$ as a function of $Q^{2}$. The red points are results extracted using the plateau method as discussed in Fig. 1 with the grey band showing the fit to the form factor in the range of $Q^{2}=$ $0-0.8 \mathrm{GeV}^{2}$ using the z-expansion $\left(\chi^{2} /\right.$ d.o.f $\left.=0.94\right)$. The blue band is the corresponding fit taking as largest $Q^{2}=0.5 \mathrm{GeV}^{2}$. The green band is the fit to the results extracted from the summation method, using $t_{s}^{\text {low }}=0.56 \mathrm{fm}$ and a maximum value of $t_{s}=1.44 \mathrm{fm}$. The strange charge factor $e_{s}=-1 / 3$ is not included.

the coefficients with $k>1$. Namely, we set $a_{k>1}=0 \pm$ $w \max \left(\left|a_{0}\right|,\left|a_{1}\right|\right)$ [24], where $w$ is a coefficient controlling the width of the Gaussian prior. We find that for $w \geq 10$ the extracted values are unaffected and therefore we set $w=10$ in the fit and use a correlated $\chi^{2}$ for the fit. The strange magnetic moment $\mu^{s} \equiv G_{M}^{s}(0)$ is then given by the fit parameter $a_{0}^{M}$. The radii are extracted from the slope of the form factors as $Q^{2} \rightarrow 0$, namely via

$$
\left\langle r_{E, M}^{2}\right\rangle^{s}=-\left.6 \frac{d G_{E, M}^{s}\left(Q^{2}\right)}{d Q^{2}}\right|_{Q^{2}=0}=\frac{-3 a_{1}^{E, M}}{2 t_{\mathrm{cut}}} .
$$

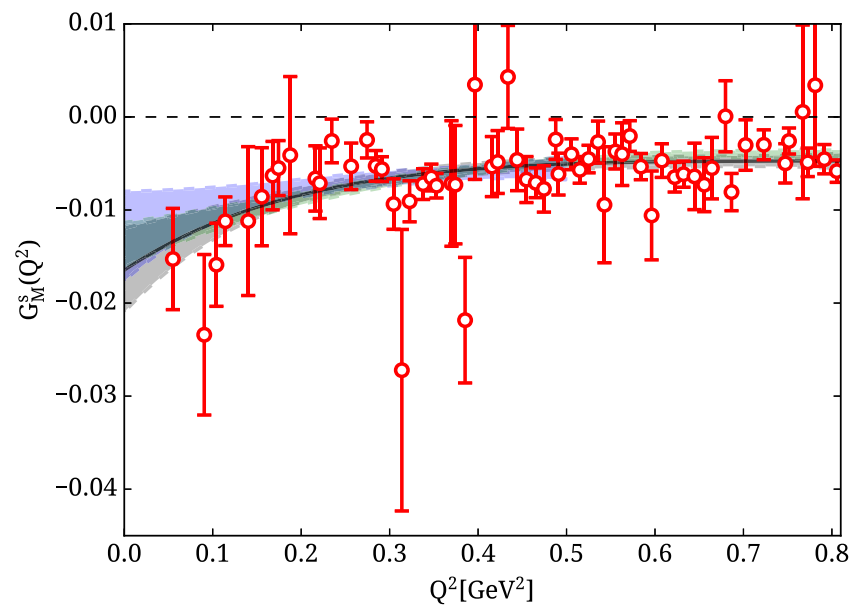

FIG. 3. $G_{M}^{s}\left(Q^{2}\right)$ as a function of $Q^{2}$. The notation is as in Fig. 2 $\left(\chi^{2} /\right.$ d.o.f $\left.=1.05\right)$.
Since the extracted quantities are computed in the limit $Q^{2} \rightarrow 0$ we provide a systematic error by comparing the maximum and minimum change in the fit parameters when we restrict the maximum value of $Q^{2}$ to $0.5 \mathrm{GeV}^{2}$.

\section{COMPARISON}

Within the twisted mass formulation we have previously analyzed an $N_{f}=2$ ensemble with close to physical pion mass, namely $m_{\pi}=130 \mathrm{MeV}$, lattice spacing $a=$ $0.094(1) \mathrm{fm}$ and lattice size $48^{3} \times 96$ [27], referred to as the cA2.09.48 ensemble. Currently, there are no other precise lattice QCD calculations of these form factors directly at the physical pion mass. The fact that we achieved the current accuracy is due to our improved methods for computing the quark loops at the physical point. Three other groups have computed the strange form factors. The analysis by the $\chi \mathrm{QCD}$ Collaboration, which included an ensemble with physical pion mass, was performed using $N_{f}=2+1$ gauge configurations of domain wall fermions (DWF) and overlap fermions for the evaluation of nucleon two- and three-point correlators. The four DWF ensembles spanned pion masses $m_{\pi} \in$ [139-330] MeV. Their final values are extracted using a chiral extrapolation since their results at the physical point alone carry larger statistical errors [25]. The other two groups used simulations with heavier than physical pions: The LHPC Collaboration analyzed one ensemble of $N_{f}=2+1$ clover-improved Wilson fermions with $m_{\pi}=317 \mathrm{MeV}$ [24]. The Mainz group [63] analyzed several ensembles of $N_{f}=2+1$

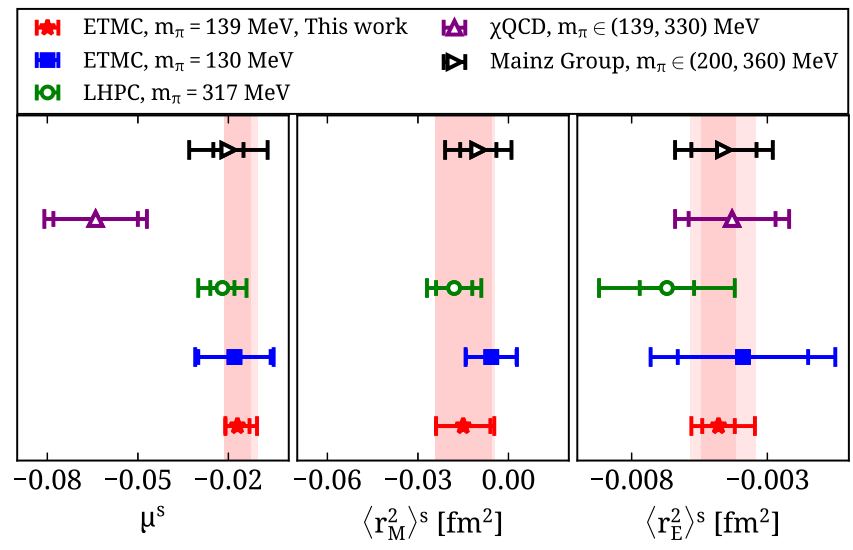

FIG. 4. Results for $\mu^{s}$ in Bohr magnetons (left), for $\left\langle r_{M}^{2}\right\rangle^{s}$ (middle) and for $\left\langle r_{E}^{2}\right\rangle^{s}$ (right). Results for the cB211.072.64 ensemble are shown with the red stars accompanied by the red error band and for the cA2.09.48 ensemble by the blue filled square [27]. Open symbols denote results extrapolated at the physical point using ensembles with larger than physical pion masses. Purple upper triangles show results from the $\chi$ QCD [25] Collaboration, black right triangles from the Mainz group [63] and green circles from the LHPC [24]. The inner error bars indicate the statistical while the outer the total, which includes systematic errors. 
$\mathcal{O}(a)$-improved Wilson fermions with pion masses $m_{\pi} \in$ [200-360] MeV and performed a chiral extrapolation to extract the value at the physical point.

In Fig. 4, we show a comparison of the magnetic moment and radii using the two twisted mass ensembles with physical pion mass to the corresponding results from the aforementioned groups. Our values for the electric radius and magnetic moment confirm a nonzero value for both. The agreement among lattice QCD results using ensembles of different values of the lattice spacings and volumes indicates that cutoff and finite volume effects are small. This is corroborated by our results using the $N_{f}=2$ cA2.09.48 ensemble with $a=0.094 \mathrm{fm}$ and the $N_{f}=2+$ $1+1 \mathrm{cB} 211.072 .64$ ensemble with $a=0.08 \mathrm{fm}$ for which no finite- $a$ dependence is observed.

\section{CONCLUSIONS}

The strange nucleon electromagnetic form factors are evaluated directly at the physical point eliminating the need for a chiral extrapolation that introduces an uncontrolled systematic error that can be large [64]. Using the model independent $\mathrm{z}$-expansion to fit the form factors we obtain the following values for the radii and magnetic moment:

$$
\begin{aligned}
\mu^{s} & =-0.017(4)\left(\begin{array}{c}
+1 \\
0
\end{array}\right)\left(\begin{array}{c}
+5 \\
0
\end{array}\right), \\
\left\langle r_{M}^{2}\right\rangle^{s} & =-0.015(9)\left(\begin{array}{l}
+3 \\
0
\end{array}\right)\left(\begin{array}{l}
+4 \\
0
\end{array}\right) \mathrm{fm}^{2}, \\
\left\langle r_{E}^{2}\right\rangle^{s} & =-0.0048(6)\left(\begin{array}{c}
0 \\
-8
\end{array}\right)\left(\begin{array}{l}
+12 \\
0
\end{array}\right) \mathrm{fm}^{2},
\end{aligned}
$$

where the first error is statistical, the second the systematic error due to excited states and the third the systematic by comparing the parameters when restricting to maximum $Q^{2}=0.5 \mathrm{GeV}^{2}$ in the fits. We obtain nonzero values for $\left\langle r_{E}^{2}\right\rangle^{s}$ and $\mu^{s}$. While the error on $\left\langle r_{M}^{2}\right\rangle^{s}$ is larger and a zero value is not excluded, all lattice QCD computations consistently predict a negative mean value. Computing these quantities to this accuracy is significant given the status of experimental searches where the results are inconclusive. For example, SAMPLE [11] finds $\mu^{s}=$ $0.37 \pm 0.20 \pm 0.26 \pm 0.15$ that is positive but also compatible with zero. More recently, the HAPPEX Collaboration finds $G_{M}^{s}=-0.070 \pm 0.067$ at $Q^{2} \sim$ $0.62 \mathrm{GeV}^{2}$ [19], while the A4 experiment [15] finds $G_{E}^{s}=$ $0.050 \pm 0.038 \pm 0.019$ and $G_{M}^{s}=0.14 \pm 0.11 \pm 0.11$ at $Q^{2}=0.22 \mathrm{GeV}^{2}$. The Q-weak experiment $[65,66]$ is aiming to measure the weak charge of the proton to unprecedented accuracy and theoretical input on the strange electromagnetic form factors can aid the interpretation of the experimental results including those from future experiments such as SoLID [67]. In addition, the P2 experiment at MESA facility at Mainz [68] targets very low $Q^{2}$ in order to improve the determination of the strange electric form factor, making our results of high relevance. In Fig. 5 we

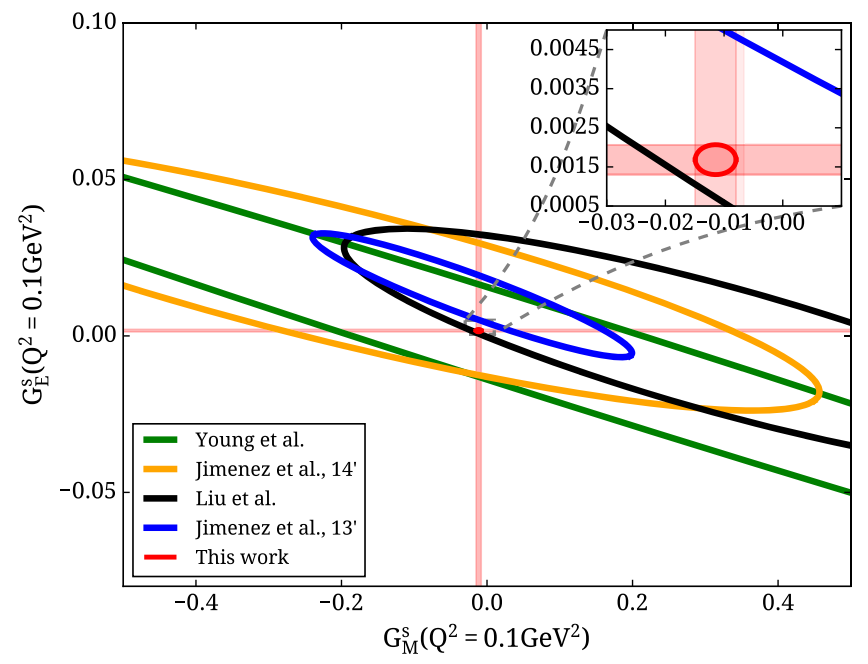

FIG. 5. The red bands show the constraints arising from the values of $G_{E}^{s}$ and $G_{M}^{s}$ at $Q^{2}=0.1 \mathrm{GeV}$ extracted in this work. The ellipses indicate $95 \%$ confidence level. The green ellipse is from Ref. [69], the orange from Ref. [22], the black from Ref. [70] and the blue from Ref. [71].

show the impact of the determination of the strange form factors on the experimental measurements at a given value of $Q^{2}=0.1 \mathrm{GeV}^{2}$. Our values provide a stringent constrain on experimental searches.

\section{ACKNOWLEDGMENTS}

We would like to thank all members of the Extended Twisted Mass Collaboration (ETMC) for a very constructive and enjoyable collaboration. M.C. acknowledges financial support by the U.S. National Science Foundation under Grant No. PHY-1714407. K. H. is financially supported by the Cyprus Research Promotion foundation under Contract No. POST-DOC/0718/0100. S. B. is supported by the Marie Skłodowska-Curie Grant Agreement No. 642069 of the European commission and from the project COMPLEMENTARY/0916/0015 funded by the Cyprus Research Promotion Foundation. The project used resources of the SuperMUC at Leibniz Supercomputing Centre under the Gauss Centre for Supercomputing e.V. project pr74yo. This work was supported by a grant from the Swiss National Supercomputing Centre (CSCS) under Project ID s702. This research uses resources of Temple University, supported in part by the National Science Foundation (Grant No. 1625061) and by the US Army Research Laboratory (Contract No. W911NF-16-2-0189). It also used XSEDE computational resources supported by National Science Foundation Grant No. TG-PHY170022 as well as on the Jureca system of the research center in Jülich, under the project ECY00. 
[1] D. B. Kaplan and A. Manohar, Nucl. Phys. B310, 527 (1988).

[2] H. Weigel, A. Abada, R. Alkofer, and H. Reinhardt, Phys. Lett. B 353, 20 (1995).

[3] V. E. Lyubovitskij, P. Wang, T. Gutsche, and A. Faessler, Phys. Rev. C 66, 055204 (2002).

[4] A. Silva, H.-C. Kim, D. Urbano, and K. Goeke, Phys. Rev. D 74, 054011 (2006).

[5] K. Goeke, H.-C. Kim, A. Silva, and D. Urbano, Eur. Phys. J. A 32, 393 (2007).

[6] R. Bijker, J. Phys. G 32, L49 (2006).

[7] P. Wang, D. B. Leinweber, and A. W. Thomas, Phys. Rev. D 89, 033008 (2014).

[8] T. J. Hobbs, M. Alberg, and G. A. Miller, Phys. Rev. C 91, 035205 (2015).

[9] T. R. Hemmert, B. Kubis, and U.-G. Meissner, Phys. Rev. C 60, 045501 (1999).

[10] J. F. Donoghue, B. R. Holstein, T. Huber, and A. Ross, Fiz. B 14, 217 (2005).

[11] D. T. Spayde et al. (SAMPLE Collaboration), Phys. Lett. B 583, 79 (2004).

[12] E. J. Beise, M. L. Pitt, and D. T. Spayde, Prog. Part. Nucl. Phys. 54, 289 (2005).

[13] F. E. Maas et al. (A4 Collaboration), Phys. Rev. Lett. 93, 022002 (2004).

[14] F. E. Maas et al., Phys. Rev. Lett. 94, 152001 (2005).

[15] S. Baunack et al., Phys. Rev. Lett. 102, 151803 (2009).

[16] K. A. Aniol et al. (HAPPEX Collaboration), Phys. Rev. Lett. 96, 022003 (2006).

[17] K. A. Aniol et al. (HAPPEX Collaboration), Phys. Lett. B 635, 275 (2006).

[18] A. Acha et al. (HAPPEX Collaboration), Phys. Rev. Lett. 98, 032301 (2007).

[19] Z. Ahmed et al. (HAPPEX Collaboration), Phys. Rev. Lett. 108, 102001 (2012).

[20] D. S. Armstrong et al. (G0 Collaboration), Phys. Rev. Lett. 95, 092001 (2005).

[21] D. Androic et al. (G0 Collaboration), Phys. Rev. Lett. 104, 012001 (2010).

[22] R. Gonzlez-Jimnez, J. A. Caballero, and T. W. Donnelly, Phys. Rev. D 90, 033002 (2014).

[23] F. E. Maas and K. D. Paschke, Prog. Part. Nucl. Phys. 95, 209 (2017).

[24] J. Green, S. Meinel, M. Engelhardt, S. Krieg, J. Laeuchli, J. Negele, K. Orginos, A. Pochinsky, and S. Syritsyn, Phys. Rev. D 92, 031501 (2015).

[25] R. S. Sufian, Y.-B. Yang, A. Alexandru, T. Draper, J. Liang, and K.-F. Liu, Phys. Rev. Lett. 118, 042001 (2017).

[26] R. S. Sufian, Y.-B. Yang, J. Liang, T. Draper, and K.-F. Liu, Phys. Rev. D 96, 114504 (2017).

[27] C. Alexandrou, M. Constantinou, K. Hadjiyiannakou, K. Jansen, C. Kallidonis, G. Koutsou, and A. V. Avilés-Casco, Phys. Rev. D 97, 094504 (2018).

[28] C. Alexandrou et al., Phys. Rev. D 98, 054518 (2018).

[29] R. Frezzotti, P. A. Grassi, S. Sint, and P. Weisz (Alpha Collaboration), J. High Energy Phys. 08 (2001) 058.

[30] R. Frezzotti and G. C. Rossi, J. High Energy Phys. 08 (2004) 007.

[31] R. Frezzotti and G. C. Rossi, J. High Energy Phys. 10 (2004) 070.
[32] B. Sheikholeslami and R. Wohlert, Nucl. Phys. B259, 572 (1985).

[33] C. Alexandrou, S. Bacchio, M. Constantinou, J. Finkenrath, K. Hadjiyiannakou, K. Jansen, G. Koutsou, and A. V. A. Casco, Phys. Rev. D 100, 014509 (2019).

[34] C. Alexandrou, S. Gusken, F. Jegerlehner, K. Schilling, and R. Sommer, Nucl. Phys. B414, 815 (1994).

[35] S. Gusken, Nucl. Phys. B, Proc. Suppl. 17, 361 (1990).

[36] M. Albanese et al. (APE Collaboration), Phys. Lett. B 192, 163 (1987).

[37] J. Foley, K. J. Juge, A. O’Cais, M. Peardon, S. M. Ryan, and J.-I. Skullerud, Comput. Phys. Commun. 172, 145 (2005).

[38] C. Alexandrou et al., arXiv:1908.10706.

[39] C. Alexandrou, K. Hadjiyiannakou, G. Koutsou, A. O'Cais, and A. Strelchenko, Comput. Phys. Commun. 183, 1215 (2012).

[40] A. Stathopoulos, J. Laeuchli, and K. Orginos, arXiv:1302 .4018 .

[41] C. Michael and C. Urbach (ETM Collaboration), Proc. Sci., LATTICE2007 (2007) 122 [arXiv:0709.4564].

[42] C. McNeile and C. Michael (UKQCD Collaboration), Phys. Rev. D 73, 074506 (2006).

[43] C. Alexandrou, M. Constantinou, V. Drach, K. Hadjiyiannakou, K. Jansen, G. Koutsou, A. Strelchenko, and A. Vaquero, Comput. Phys. Commun. 185, 1370 (2014).

[44] A. Abdel-Rehim, C. Alexandrou, M. Constantinou, V. Drach, K. Hadjiyiannakou, K. Jansen, G. Koutsou, and A. Vaquero, Phys. Rev. D 89, 034501 (2014).

[45] R. Babich, M. A. Clark, and B. Joo, in SC 10 (Supercomputing 2010) New Orleans, Louisiana (2010), http://www1.jlab.org/U1/publications/view_pub.cfm?pub_id= 10186.

[46] M. A. Clark, B. Jo, A. Strelchenko, M. Cheng, A. Gambhir, and R. Brower, arXiv:1612.07873.

[47] C. Alexandrou, K. Hadjiyiannakou, G. Koutsou, A. Strelchenko, and A. V. Avils-Casco, Proc. Sci., LATTICE2013 (2014) 411 [arXiv:1401.6750].

[48] C. Alexandrou, M. Constantinou, S. Dinter, V. Drach, K. Jansen, C. Kallidonis, and G. Koutsou, Phys. Rev. D 88, 014509 (2013).

[49] C. Alexandrou, M. Brinet, J. Carbonell, M. Constantinou, P. A. Harraud, P. Guichon, K. Jansen, T. Korzec, and M. Papinutto, Phys. Rev. D 83, 094502 (2011).

[50] C. Alexandrou, G. Koutsou, J. W. Negele, and A. Tsapalis, Phys. Rev. D 74, 034508 (2006).

[51] C. Alexandrou, S. Bacchio, M. Constantinou, K. Hadjiyiannakou, K. Jansen, G. Koutsou, and A. V. Aviles-Casco, arXiv:1909.00485.

[52] G. Martinelli and C. T. Sachrajda, Phys. Lett. B 196, 184 (1987).

[53] G. Martinelli, C. Pittori, C. T. Sachrajda, M. Testa, and A. Vladikas, Nucl. Phys. B445, 81 (1995).

[54] M. Göckeler, R. Horsley, H. Oelrich, H. Perlt, D. Petters, P. E. L. Rakow, A. Schäfer, G. Schierholz, and A. Schiller, Nucl. Phys. B544, 699 (1999).

[55] C. Alexandrou, M. Constantinou, T. Korzec, H. Panagopoulos, and F. Stylianou (ETM Collaboration), Phys. Rev. D 83, 014503 (2011). 
[56] C. Alexandrou, M. Constantinou, T. Korzec, H. Panagopoulos, and F. Stylianou, Phys. Rev. D 86, 014505 (2012).

[57] C. Alexandrou, M. Constantinou, and H. Panagopoulos (ETM Collaboration), Phys. Rev. D 95, 034505 (2017).

[58] M. Constantinou et al. (ETM Collaboration), J. High Energy Phys. 08 (2010) 068.

[59] M. Constantinou, R. Horsley, H. Panagopoulos, H. Perlt, P. E. L. Rakow, G. Schierholz, A. Schiller, and J. M. Zanotti, Phys. Rev. D 91, 014502 (2015).

[60] B. Bhattacharya, R. J. Hill, and G. Paz, Phys. Rev. D 84, 073006 (2011).

[61] R. J. Hill and G. Paz, Phys. Rev. Lett. 107, 160402 (2011).

[62] R. J. Hill and G. Paz, Phys. Rev. D 82, 113005 (2010).

[63] D. Djukanovic, K. Ottnad, J. Wilhelm, and H. Wittig, Phys. Rev. Lett. 123, 212001 (2019).
[64] G. Colangelo, U. Wenger, and J. M. S. Wu, Phys. Rev. D 82, 034502 (2010).

[65] D. S. Armstrong et al., arXiv:1202.1255.

[66] D. Androi et al. (Qweak Collaboration), Nature (London) 557, 207 (2018).

[67] Y.X. Zhao (SoLID Collaboration), in 22nd International Symposium on Spin Physics (SPIN 2016) Urbana, IL, USA, 2016 (2017).

[68] D. Becker et al., Eur. Phys. J. A 54, 208 (2018).

[69] R. D. Young, J. Roche, R. D. Carlini, and A. W. Thomas, Phys. Rev. Lett. 97, 102002 (2006).

[70] J. Liu, R. D. McKeown, and M. J. Ramsey-Musolf, Phys. Rev. C 76, 025202 (2007).

[71] R. Gonzalez-Jimenez, J. A. Caballero, and T. W. Donnelly, Phys. Rep. 524, 1 (2013). 